\title{
Perfil epidemiológico de casos de surtos de doenças transmitidas por alimentos ocorridos no Paraná, Brasil
}

\section{Epidemiological profile of foodborne diseases outbreaks occurred in Parana state, Brazil}

\author{
Jonatas Campos de Almeida ${ }^{1}$, Caroline Marie Sundin de Paula ${ }^{2}$, Walfrido \\ Kuhl Svoboda ${ }^{3}$, Márcia Oliveira Lopes ${ }^{4}$, Marcelo Pilonetto Pilonetto ${ }^{5}$, Wanda \\ Moscalewski Abrahão ${ }^{6}$, Eliane Carneiro Gomes ${ }^{7}$
}

\begin{abstract}
Resumo
A investigação dos surtos de Doenças Transmitidas por Alimentos (DTA) é desafiadora. Para a Vigilância em Saúde é fundamental a identificação de grupos e fatores de risco associados às DTA. O objetivo deste trabalho foi caracterizar o perfil epidemiológico dos surtos de DTA ocorridos nos 29 municípios que compõe a 2 $2^{\text {a }}$ egional de Saúde, da Secretaria Estadual de Saúde do Paraná, Brasil, entre 20052008. Tabularam-se 472 laudos de alimentos do Laboratório Central do Estado do Paraná (LACEN-PR) e fichas notificadas no Sistema de Informação de Agravos de Notificação (SINAN) de casos suspeitos de DTA. O grupo mais acometido foi de indivíduos do sexo feminino com 50,51\% dos casos. Indivíduos entre 20 a 49 anos de idade foram os mais frequentemente envolvidos com $49,50 \%$ das ocorrências. Restaurantes $(27,65 \%)$ e residências dos indivíduos acometidos $(36,17 \%)$ foram os locais de preparo e consumo mais frequentemente envolvidos. Manipulação/preparo inadequados dos alimentos (36,95\%) e forma de consumo inadequada $(23,90 \%)$ foram os fatores causais mais predominantes. A maior parte das confirmações dos surtos fundamentou-se em achados clínico-epidemiológicos $(44,68 \%)$. Com relação aos micro-organismos identificados nas amostras analisadas, os resultados apontaram predominância Escherichia coli (indicador sanitário), seguido pelo Bacillus cereus e Staphyloccus aureus. Os alimentos mais frequentemente envolvidos foram o leite $(29,68 \%)$ e as frutas, verduras e legumes $(24,21 \%)$. Com a pesquisa é possível orientar medidas preventivas e de controle de DTA na população pesquisada.

Palavras-chaves: Alimentos. Surtos de Doenças. Doenças transmitidas por alimentos.
\end{abstract}

${ }^{1}$ Laboratório de Zoonoses e Saúde Pública, Departamento de Medicina Veterinária Preventiva, Universidade Estadual de Londrina (UEL). Londrina, Paraná, Brasil. E-mail: jonatas_campos86@hotmail.com.

${ }^{2}$ Curso de Medicina Veterinária, Universidade Federal do Paraná (UFPR), Curitiba, Paraná. E-mail: carol_marie87@yahoo.com.br.

${ }^{3}$ Professor adjunto do Departamento de Saúde Comunitária da UFPR, Programa de Pós-Graduação em Ciências Farmacêuticas, Departamento de Saúde Comunitária, Laboratório de Saúde Pública e Saúde Ambiental, Universidade Federal do Paraná (UFPR), Curitiba, Paraná. E-mail: walfrido.ufpr@gmail.com.

${ }^{4}$ Professora Assistente do Departamento de Saúde Comunitária da UFPR. Servidora do Laboratório Central do Estado do Paraná, Secretaria Estadual de Saúde do Paraná, Curitiba, Paraná. E-mail: marciaoliveira.ufpr@gmail.com.

${ }^{5}$ Servidor do Laboratório Central do Estado do Paraná, Secretaria Estadual de Saúde do Paraná, Curitiba, Paraná. E-mail: marcelopilonetto@gmail.com.

${ }^{6}$ Professora Assistente do Departamento de Farmácia da UFPR, Servidora do Laboratório Central do Estado do Paraná, Secretaria Estadual de Saúde do Paraná, Curitiba, Paraná. E-mail: wanda.abrahao@terra.com.br.

${ }^{7}$ Professora Associada do Departamento de Saúde Comunitária da UFPR, Programa de Pós-Graduação em Ciências Farmacêuticas. Contato principal para correspondências. E-mail: ecarneirogomes@gmail.com. 


\begin{abstract}
The investigation of outbreaks of Food Borne Diseases (FBD) is challenging. For the Health Surveillance is essential to identify groups and risk factors associated with the DTA. The aim of this study was to characterize the epidemiology of outbreaks of DTA occurred in 29 counties that comprise the 2nd Regional Health, the Department of Health of Paraná, Brazil, from 2005-2008. Tabulated to 472 reports of food the Central Laboratory of the State of Paraná (LACEN-PR) chips and notified the Information System for Notifiable Diseases (SINAN) of suspected cases of DTA. The group most affected were females with $50.51 \%$ of the cases. Individuals between 20 and 49 years of age were most often involved in $49.50 \%$ of cases. Restaurants $(27.65 \%)$ of affected individuals and households $(36.17 \%)$ were the preparation and consumption sites most frequently involved. Manipulation / inadequate preparation of food $(36.95 \%)$ and inadequate form of consumption $(23.90 \%)$ were the most prevalent causative factors. Most confirmations of outbreaks was based on clinical and epidemiological (44.68\%). With respect to micro-organisms identified in the samples analyzed, the results showed predominance Escherichia coli (health indicator), followed by Bacillus cereus and Staphylococcus aureus. The foods most frequently involved were milk (29.68\%) and fruits and vegetables (24.21\%). With this study can guide preventive measures and control of DTA in the population studied.
\end{abstract}

Keywords: Food. Disease Outbreaks. Foodborne Diseases.

\section{Introdução}

O termo Doenças Transmitidas por Alimentos (DTA) é genérico, sendo utilizado para síndromes, geralmente acompanhas por: anorexia, náuseas, vômitos e/ou diarréia. As DTA são atribuídas à ingestão de alimentos ou água contaminados por bactérias, vírus, parasitas, toxinas, príons, agrotóxicos, produtos químicos e metais pesados (BRASIL, 2005). Além dos sintomas de caráter gastrointestinal, podem ocorrer sinais em diferentes órgãos e sistemas, como por exemplo: meninges, rins, fígado, sistema nervoso central, terminações nervosas periféricas e outros, conforme o agente etiológico envolvido (OPAS, 2001).

As DTA, podem se manifestar na forma de: infecções, intoxicações e toxinfecções alimentares. As infecções alimentares são resultantes da ingestão de alimentos que contém micro-organismos patogênicos vivos. As intoxicações alimentares ocorrem quando as toxinas das bactérias ou fungos estão presentes no alimento ingerido. As toxinas podem estar presentes naturalmente no alimento, como no caso de alguns fungos ou peixes. As toxinfecções alimentares resultam da ingestão de alimentos com certa quantidade de microorganismos causadores de doenças, capazes de produzir ou liberar toxinas após serem ingeridos (OPAS, 2001).

Caracteriza-se como um surto de DTA o episódio em que duas ou mais pessoas apresentam doença semelhante após a ingestão de alimentos, inclusive água, de mesma origem e onde a evidência epidemiológica ou análise laboratorial apontam os alimentos e/ou água como veículos da doença (OPAS, 2001). Para a descrição de um surto de DTA, determinados fatores devem ser considerados: a situação, o número de pessoas afetadas, o índice de ataque por idade, sexo e raça, o número de pessoas que não foram atingidas, o agente e o período de incubação, a natureza clínica da doença, o veículo alimentar e o modo de transmissão para os alimentos e para as vítimas (HOBBS; ROBERTS, 1999).

O número de casos de DTA é difícil de estimar, mas foi relatado que em 2005 cerca de 1,8 milhões de pessoas morreram por doenças diarreicas. Uma grande proporção desses casos pode ser atribuída à contaminação de alimento e água de bebida. A alta incidência de doenças diarreicas em diversos países em desenvolvimento sugere maior atenção a questões de sanidade dos alimentos. Identificar a doença e quais os fatores que contribuíram para sua 
ocorrência é uma prioridade na investigação. Surto com causa ignorada tem geralmente como causa a notificação tardia, a ausência de coleta de amostras clínicas e de alimentos em tempo adequado, ou testes laboratoriais inadequados (EDUARDO; KATSUYA; BASSIT, 2003).

Conforme dados divulgados pelo Sistema Único de Saúde (SUS), no Estado do Paraná, no ano de 2000, o custo médio por internação foi de R\$ 471,59. Neste mesmo período, ocorreram 219 surtos de DTAs, nos quais 1000 pessoas foram hospitalizadas e, estima-se que 8.663 ficaram doentes. Desse modo, pode-se estimar que no ano de 2000 o governo gastou $\mathrm{R} \$ 1.870 .000,00$ somente com internações devido às doenças transmitidas por alimentos (BRASIL, 2005).

A investigação de um surto de DTA se embasa em três eixos principais: (1) a investigação epidemiológica propriamente dita, por meio de formulários com entrevistas aos envolvidos no surto, para identificar o veículo de transmissão e o provável agente etiológico; (2) a investigação laboratorial, com a coleta de amostras clínicas de pacientes, alimentos e água, para confirmação e (3) a investigação ambiental, ou seja, averiguação do local de ocorrência do surto para se detectar os fatores contribuintes que possibilitaram o surgimento do mesmo (SANTA CATARINA, 2006).

Nesse contexto a Vigilância Sanitária atua com medidas capazes de eliminar, diminuir ou prevenir riscos e agravos à saúde individual e coletiva. Exerce fiscalização e controle sobre o meio ambiente, detectando falha no controle do fluxograma de produção (riscos biológicos, químicos ou físicos), identificando os seus pontos críticos e educando com vistas à transformação nos procedimentos de preparo e produção dos alimentos, higiene dos manipuladores e da população (BRASIL, 1994).

Neste estudo objetivou-se caracterizar o perfil epidemiológico dos surtos de DTA ocorridos na Região Metropolitana de Curitiba, no Estado do Paraná, Brasil, no período de 2005 a 2008.

\section{Material e Métodos}

A pesquisa se desenvolveu nos 29 municípios que compõe a área de abrangência da $2^{\text {a }}$ Regional de Saúde, da Secretaria Estadual de Saúde do Estado do Paraná, Brasil (Figuras 1 e 2), englobando os casos de surtos alimentares registrados dentro do período compreendido entre os anos de 2005 a 2008.

Figuras 1 - Mapa das 22 Regionais de Saúde do Estado do Paraná, Brasil.

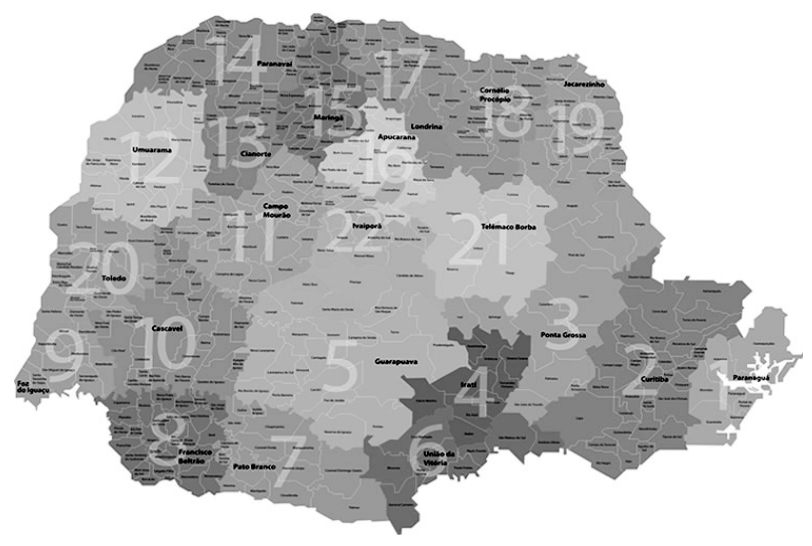

Fonte: PARANÁ, 2012a.

Figuras 2 - $2^{\text {a }}$ Regional de Saúde do Estado do Paraná

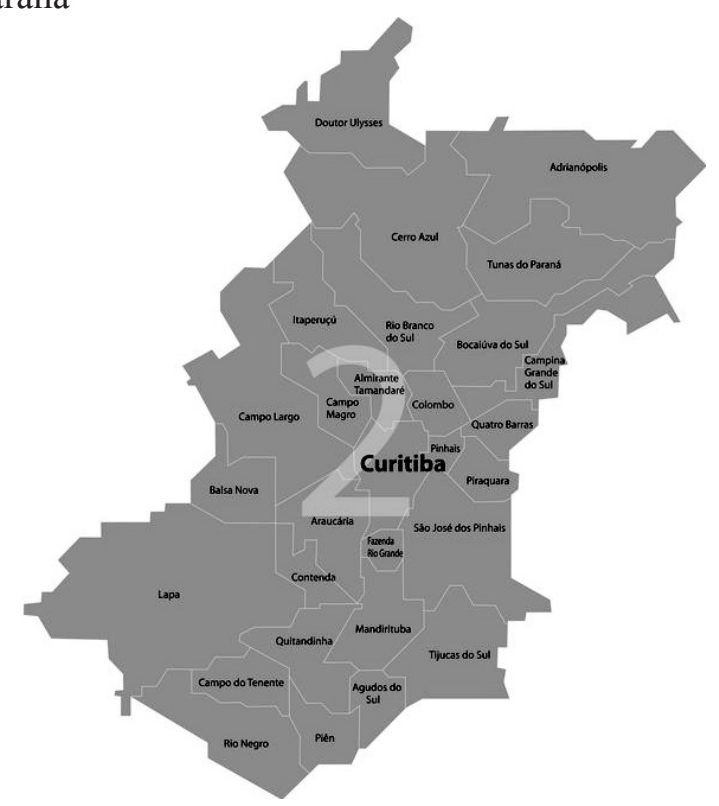

Fonte: PARANÁ, 2012b. 
Tabularam-se 472 laudos de resultados de análise de alimentos emitidos pelo Laboratório Central do Estado do Paraná (LACEN-PR) e 47 fichas de investigação de surtos de DTA notificados no Sistema de Informação de Agravos de Notificação (SINAN), no período analisado.

Foram tabulados dos laudos de análise de alimentos do LACEN-PR dados referentes a: i) número do laudo, ii) alimento envolvido, iii) município de ocorrência, iv) data de coleta de material, v) data do resultado, vi) resultado da análise em relação ao agente etiológico isolado.

Nas fichas de notificação do SINAN os dados levantados referiam-se a: sexo, faixa etária, sintomas, locais de preparo e consumo do alimento envolvido, fatores causais, critérios de confirmação, agentes etiológicos envolvidos, municípios de notificação.

\section{Resultados e Discussão}

No período estudado foram analisados 47 surtos de DTA ocorridos na $2^{\text {a }}$ RS. Observou-se que em 2005 e 2008, o município de Curitiba foi o único a apresentar as notificações dos surtos, correspondendo a 27 e 11 amostras com resultado positivo respectivamente em relação ao agente etiológico isolado. Esse dado é de extrema importância, pois pode ser compreendido como uma situação de subnotificação pelos outros municípios pertencentes à $2^{\mathrm{a}} \mathrm{RS}$. No ano de 2006, 57,5\% (27/47) das amostras positivas pertenciam ao município de Curitiba, 12,5\% (6/47) a Rio Negro, 7,5\% (4/47) a Campo Largo, 7,5\% (4/47) a Piraquara, 5\% (2/47) a Lapa e 2,5\% (1/47) para cada um dos municípios relacionados a seguir: Araucária, Almirante Tamandaré, Campina Grande do Sul e Itaperuçu. Em relação ao ano de 2007 a distribuição de casos positivos foi de 88,23\% (19/21) em Curitiba e 5,88\% (1/21) para Campina Grande do Sul e 5,88\% (1/21) para Campo Largo. Observa-se que o município de Curitiba, no período envolvido pela pesquisa, ficou responsável por 79,24\% (84/106) dos casos notificados.

Em relação ao gênero, o grupo mais acometido foram indivíduos do sexo feminino, respondendo por 50,51\% (246/487) dos casos notificados (Figura 3). Quanto à faixa etária, indivíduos entre $20-\mid$ 49 anos de idade foram os mais frequentemente envolvidos com 49,50\% (241/487) das ocorrências (Figura 4).

Figura 3 - Número total de indivíduos envolvidos em surtos por Doenças Transmitidas por Alimentos, conforme gênero, na $2^{\mathrm{a}} \mathrm{RS}$ do Estado do Paraná, Brasil, no período de 2005-2008.

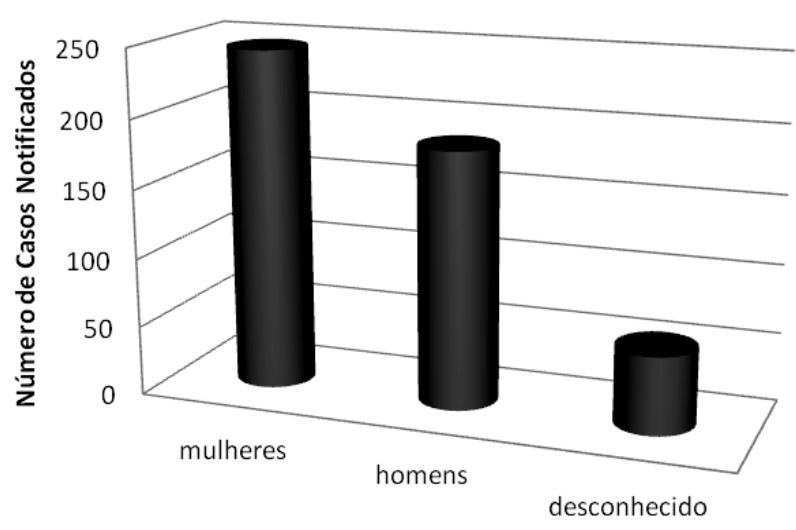

Fonte: Autores.

Figura 4 - Número total de indivíduos envolvidos em surtos por Doenças Transmitidas por Alimentos, de acordo com a faixa etária, na $2^{\text {a }} \mathrm{RS}$ do Estado do Paraná, no período de 2005-2008.

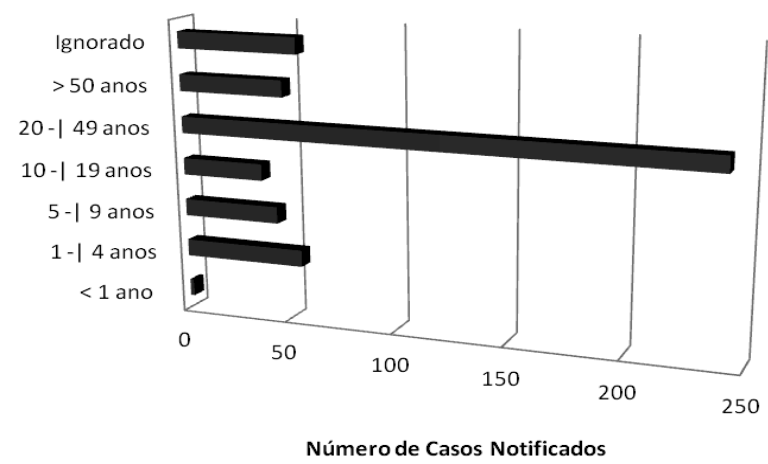

Fonte: Autores. 
Quanto ao local de preparo do alimento envolvido no surto, $27,7 \%$ (13/47) eram oriundos de restaurantes e $25,5 \%(12 / 47)$ da residência do indivíduo afetado (Tabela 1).

Tabela 1 - Percentual de distribuição das ocorrências dos surtos de Doenças Transmitidas por Alimentos, de acordo com o local de preparo dos alimentos, na $2^{\text {a }}$ RS do Estado do Paraná, Brasil, no período de 2005 a 2008 .

\begin{tabular}{lcc}
\hline Local de preparação & $\begin{array}{c}\text { Número de } \\
\text { Notificação }\end{array}$ & Percentual (\%) \\
\hline Restaurante & 13 & 27,66 \\
Residência & 12 & 25,53 \\
Outros & 7 & 14,89 \\
Indústria & 5 & 10,64 \\
Ignorados & 5 & 10,64 \\
LanchonetelConfeitaria Padaria & 3 & 6,38 \\
Crechelescola & 1 & 2,13 \\
Refeitório & 1 & 2,13 \\
TOTAL & 47 & 100 \\
\hline
\end{tabular}

Fonte: Autores.

Contudo o principal local de ingestão do alimento contaminado foi a residência do indivíduo acometido, responsável por 36,17\% (17/47) das notificações seguido dos restaurantes com 34,04\% (16/47) dos casos (Tabela 2). Episódios de surtos de DTA ocorrem dentro das próprias residências dos indivíduos envolvidos, seja por erros na conservação dos alimentos, ou mesmo, nas boas práticas de preparo (LOPES, 2007). De acordo com dados de Paraná (2011), 47,7\% das situações notificadas quanto ao local de preparo e de ingestão do alimento envolvido no surto foram as residências. 
Tabela 2 - Distribuição das ocorrências dos surtos de Doenças Transmitidas por Alimentos, de acordo com o local de ingestão dos alimentos, na 2a RS do Estado do Paraná, Brasil, no período de 2005 a 2008.

\begin{tabular}{lcc}
\hline Local de preparação & $\begin{array}{c}\text { Número de } \\
\text { Notificação }\end{array}$ & Percentual (\%) \\
\hline Residência & 17 & 36,17 \\
Restaurante & 16 & 34,04 \\
Crechelescola & 5 & 10,64 \\
Outros & 4 & 8,51 \\
Refeitório & 3 & 6,38 \\
LanchonetelConfeitaria Padaria & 2 & 4,25 \\
TOTAL & 47 & 100 \\
\hline
\end{tabular}

Fonte: Autores.

Na distribuição dos fatores causais de DTA, (11/46) dos surtos (Tabela 3). Esses dados refletem observou-se que o fator predominante foi a a necessidade de medidas de educação e higiene, manipulação/preparo inadequados dos alimentos, não apenas para aqueles que estarão envolvidos na com 36,96\% (17/46) das ocorrências e a forma cadeia de produção de um alimento, como também de conservação inadequada reportada em 23,91\% do próprio consumidor. (PARANÁ, 2011).

Tabela 3 - Distribuição dos fatores causais de Doenças Transmitidas por Alimentos, na 2 $2^{\mathrm{a}}$ Regional de Saúde do Estado do Paraná, Brasil, no período de 2005

\begin{tabular}{lcc}
\hline Fatores causais & $\begin{array}{c}\text { Número de } \\
\text { Notificação }\end{array}$ & Percentual(\%) \\
\hline Manipulação preparação & 17 & 36,96 \\
inadequada & & \\
Conservação inadequada & 11 & 23,91 \\
Matéria-prima imprópria & 8 & 17,39 \\
Outros & 10 & 21,74 \\
TOTAL & 46 & 100 \\
\hline
\end{tabular}

Fonte: Autores. 
Dentre os fatores que mais contribuíram para a ocorrência de surtos de DTA descritos por Amson et al. (2006), no Estado do Paraná, entre 1978 e 2000, a matéria-prima contaminada antes do preparo $(75,8 \%)$ e a matéria-prima contaminada por bactérias durante o preparo envolvendo os manipuladores $(44,2 \%)$ foram as mais reportadas. Os manipuladores de alimentos são de grande importância em surtos de DTA, uma vez que podem albergar agentes como a $E$. coli e o $S$. aureus. As mãos dos manipuladores de alimentos podem, pelo contato direto ou por perdigotos (gotículas), manter a cadeia epidemiológica da intoxicação alimentar estafilocócica (LARIA; FURLANETTO; CAMPOS, 1980; SNYDER, 1992).

$\mathrm{O}$ processamento inadequado do alimento por meio do calor $(38,3 \%)$ e o reaquecimento feito de forma inadequada $(23,4 \%)$ se correlacionam com a sobrevivência dos micro-organismos, além da conservação inadequada pelo frio $(88,5 \%)$ e o intervalo de tempo longo entre o preparo e o consumo implica na multiplicação bacteriana (90,6\%). A porcentagem dos fatores contribuintes para os surtos, notoriamente excede $100 \%$ devido ao fato de que há uma interação entre diversos desses fatores, concomitantemente para cada surto (AMSON; HARACEMIV; MASSON, 2006).

Os critérios de confirmação dos casos de surtos de DTA basearam-se em achados clínicosepidemiológicos $(44,68 \% ; 21 / 47)$ e laboratorial $(23,40 \% ; 11 / 47)$. Conforme dados publicados por Amson, Haracemiv e Masson (2006), no período entre 1978 - 2000 no Estado do Paraná, 41,72\% dos surtos foram confirmados por análise laboratorial. Paraná (2011) revela que $39,85 \%$ dos achados em surtos de DTA se baseavam em exames laboratoriais e 29,25\% em exames clínico-epidemiológicos.

Com relação aos agentes etiológicos mais frequentes em amostras positivas os resultados no ano de 2005, foram 27 amostras alimentares positivas, com 51,85\% (14/27) de positividade do indicador sanitário Escherichia coli, 29,62\%
(8/27) para Bacillus cereus, 11,11\% (3/27) para Staphylococcus aureus e 7,40\% (2/27) para Salmonella sp. Já no ano de 2006, foram diagnosticadas 47 amostras positivas, sendo $37,5 \%$ (18/47) para E.coli, 31,25\% (15/47) para B.cereus, $18,75 \%(9 / 47)$ para S.aureus e 10,41\% (5/47) para Salmonella sp. No ano de 2007, foram encontradas 21 amostras positivas, sendo 78,94\% (15/21) para E. coli, 21,05\% (4/21) para B.cereus, 5,26\% (1/21) para S.aureus e 5,26\% (1/21) para Pseudomonas aeruginosa. Por fim, no ano de 2008, foram encontradas 11 amostras positivas, sendo $45,45 \%$ (5/11) para B.cereus, 27,27\% (3/11) para E.coli, $18,18 \%$ (2/11) para S.aureus e 9,1\% (1/11) para Clostrídios Sulfito Redutores (CSR).

De acordo com Amson, Haracemiv e Masson (2006), no Estado do Paraná entre o período de 1978 e 2000, os agentes bacterianos estiveram envolvidos representaram $68,01 \%$ dos surtos, sendo confirmados em $35,64 \%$ destes. Entre os casos confirmados, 56,95\% eram S.aureus, 24,93\% Salmonella spp., 2,9\% Clostridium perfringens, $2,62 \%$ B.cereus, $6,04 \%$ E. coli e 3,7\% Shiguella spp. Estes dados diferem daqueles encontrados na nossa pesquisa, onde a predominância foi de casos pela enterobactéria E.coli seguida pela bactéria formadora de esporos, B.cereus.

Os alimentos mais frequentes envolvidos em surtos de DTA correlacionado ao agente etiológico apontou para os alimentos com maior número de casos positivos foram o leite $(30,40 \%)$ com Staphylococcus aureus, as frutas, vegetais e cereais $(24,80 \%)$ com Bacillus cereus, a carne de frango $(12,8 \%)$ com Clostridium perfringens e os ovos (11,20\%) com Staphylococcus aureus (Tabela 4). 
Tabela 4 - Distribuição das ocorrências dos surtos de Doenças Transmitidas por Alimentos, de acordo com o tipo de alimento e agente etiológico encontrado, na $2^{\mathrm{a}} \mathrm{RS}$ do Estado do Paraná, Brasil, no período de 2005 a 2008.

\begin{tabular}{|c|c|c|c|c|c|c|c|c|c|}
\hline $\begin{array}{c}\text { AGENTE } \\
\text { ETIOLÓGICO }\end{array}$ & $\begin{array}{c}\text { CARNE } \\
\text { BOVINA }\end{array}$ & $\begin{array}{l}\text { CARNE } \\
\text { SUINAA }\end{array}$ & $\begin{array}{c}C A R N E \\
D E \\
F R A N G O\end{array}$ & $\begin{array}{c}C A R N E \\
D E \\
P E D X E\end{array}$ & LEITE & ovos & $\begin{array}{c}\text { FRUTAS, } \\
\text { VEGETAIS } \\
E \\
\text { CEREAIS }\end{array}$ & OUTROS & TOTAL \\
\hline Bacillus cereus & $\begin{array}{c}11.76 \% \\
(4 / 34)\end{array}$ & - & $\begin{array}{c}11.76 \% \\
(4 / 34)\end{array}$ & $\begin{array}{l}2.95 \% \\
(1 / 34)\end{array}$ & $\begin{array}{c}26.47 \% \\
(9 / 34)\end{array}$ & $\begin{array}{l}5.88 \% \\
(2 / 34)\end{array}$ & $\begin{array}{l}29.41 \% \\
(10 / 34)\end{array}$ & $\begin{array}{c}11.76 \% \\
(4 / 34)\end{array}$ & $\begin{array}{c}100 \% \\
(34 / 34)\end{array}$ \\
\hline Escherichia. coli & $\begin{array}{l}10.81 \\
(4 / 37)\end{array}$ & $\begin{array}{l}2.7 \% \\
(1 / 37)\end{array}$ & $\begin{array}{l}13.51 \% \\
(5 / 37)\end{array}$ & - & $\begin{array}{c}24.33 \% \\
(9 / 37)\end{array}$ & $\begin{array}{l}8.1 \% \\
(3 / 37)\end{array}$ & $\begin{array}{l}35.13 \% \\
(13 / 37)\end{array}$ & $\begin{array}{l}5.4 \% \\
(2 / 37)\end{array}$ & $\begin{array}{c}100 \% \\
(37 / 37)\end{array}$ \\
\hline $\begin{array}{l}\text { Clostridium } \\
\text { perfringens }\end{array}$ & - & - & $\begin{array}{c}100 \% \\
(2 / 2)\end{array}$ & - & - & - & - & - & $\begin{array}{l}100 \% \\
(2 / 2)\end{array}$ \\
\hline $\begin{array}{c}\text { Coliformes } \\
\text { C) }\end{array}$ & $\begin{array}{c}10 \% \\
(2 / 20)\end{array}$ & $\begin{array}{c}5 \% \\
(1 / 20)\end{array}$ & $\begin{array}{c}10 \% \\
(2 / 20)\end{array}$ & - & $\begin{array}{c}40 \% \\
(8 / 20)\end{array}$ & $\begin{array}{c}15 \% \\
(3 / 20)\end{array}$ & $\begin{array}{c}15 \% \\
(3 / 20)\end{array}$ & $\begin{array}{c}5 \% \\
(1 / 20)\end{array}$ & $\begin{array}{c}100 \% \\
(20 / 20)\end{array}$ \\
\hline Salmonella sp. & $\begin{array}{c}14.28 \% \\
(1 / 7)\end{array}$ & - & $\begin{array}{c}14.28 \% \\
(1 / 7)\end{array}$ & - & $\begin{array}{c}28.58 \% \\
(2 / 7)\end{array}$ & $\begin{array}{c}14.28 \% \\
(1 / 7)\end{array}$ & $\begin{array}{c}14.28 \% \\
(1 / 7)\end{array}$ & $\begin{array}{c}14.28 \% \\
(1 / 7)\end{array}$ & $\begin{array}{l}100 \% \\
(7 / 7)\end{array}$ \\
\hline $\operatorname{CSR} *$ & - & $\begin{array}{l}50 \% \\
(2 / 4)\end{array}$ & - & - & $\begin{array}{l}50 \% \\
(2 / 4)\end{array}$ & - & - & - & $\begin{array}{l}100 \% \\
(4 / 4)\end{array}$ \\
\hline $\begin{array}{c}\text { Staphylococcus } \\
\text { aureus }\end{array}$ & $\begin{array}{l}4.76 \% \\
(1 / 21)\end{array}$ & $\begin{array}{l}4.76 \% \\
(1 / 21)\end{array}$ & $\begin{array}{l}9.52 \% \\
(2 / 21)\end{array}$ & - & $\begin{array}{c}38.09 \% \\
(8 / 21)\end{array}$ & $\begin{array}{c}23.80 \% \\
(5 / 21)\end{array}$ & $\begin{array}{c}19.04 \% \\
(4 / 21)\end{array}$ & - & $\begin{array}{c}100 \% \\
(21 / 21)\end{array}$ \\
\hline TOTAL & $\begin{array}{c}9.6 \% \\
(12 / 125)\end{array}$ & $\begin{array}{c}4 \% \\
(5 / 125)\end{array}$ & $\begin{array}{c}12.8 \% \\
(16 / 125)\end{array}$ & $\begin{array}{c}0,8 \% \\
(1 / 125)\end{array}$ & $\begin{array}{c}30.4 \% \\
(38 / 125)\end{array}$ & $\begin{array}{c}11.20 \% \\
(14 / 125)\end{array}$ & $\begin{array}{c}24.80 \\
(31 / 125)\end{array}$ & $\begin{array}{c}6.4 \% \\
(8 / 125)\end{array}$ & $\begin{array}{c}100 \% \\
(125 / 125)\end{array}$ \\
\hline
\end{tabular}

Fonte: Autores. 
Os dados encontrados durante a presente pesquisa evidenciam os produtos de origem animal como os principais envolvidos em surtos de DTA (leite, ovos e carne de aves, liderando a casuística), mas também encontra nos produtos de origem vegetal uma potencial fonte de contaminação.

De acordo com Alcocer (2004), no Paraná, os produtos à base de ovos foram associados a $47,4 \%$ dos referidos surtos, evidenciando o risco potencial que esse alimento pode representar para a saúde pública. O mesmo autor ainda relata que dos 217 surtos de salmonelose ocorridos no período entre 1999 a 2004, o sorovar Enteritidis foi identificado em $87,0 \%$ e $89,8 \%$ das cepas isoladas de pacientes e de alimentos envolvidos nesses surtos, respectivamente. Silva, Ramalho e Figueiredo (2004) relatam que, em Maceió/AL, a Salmonella foi o micro-organismo identificado em $54,2 \%$ dos casos de surtos de origem alimentar, e a grande maioria dos alimentos preparados que estavam contaminados foram aqueles em que o ovo foi utilizado cru.

A falta de programas de educação no que se refere a segurança alimentar dirigidos à população certamente está relacionada com a realidade encontrada. A maioria dos consumidores desconhece os requisitos necessários para a adequada manipulação de alimentos e, principalmente, desconhece os riscos que podem estar associados a alimentos contaminados (AMSON; HARACEMIV; MASSON, 2006). A alimentação de acordo com os padrões higiênicos satisfatórios é condição fundamental para a promoção e manutenção da saúde. E alimentos (SOARES et al., 2006).

\section{Conclusões}

O estudo dos 47 surtos de DTA detectados na $2^{\mathrm{a}} \mathrm{RS}$ no período estudado (2005 e 2008) demonstrou que o serviço de Vigilância em Saúde do município de Curitiba - PR foi o principal notificador, evidenciando a subnotificação dos demais municípios da região metropolitana de Curitiba - PR.

A análise demonstrou que em relação ao gênero, o grupo mais acometido foi de indivíduos do sexo feminino, com maior frequência na faixa etária de 20 a 49 anos. Os restaurantes e residências dos indivíduos afetados foram os locais de preparo mais envolvidos. Sendo a residência o principal local de ingestão do alimento contaminado. O fator causal predominante constatado foi a manipulação/ preparo inadequados dos alimentos, seguido pela conservação inadequada dos mesmos demonstrando necessidade de implementação das ações educativas voltadas a manipuladores e consumidores.

Com relação aos agentes etiológicos mais frequentes nas amostras positivas os resultados evidenciaram o indicador sanitário Escherichia coli, Bacillus cereus, Staphylococcus aureus e Salmonella sp.,sendo que os alimentos mais frequentemente envolvidos em surtos de DTA foram o leite, as frutas, vegetais e cereais, a carne de frango e os ovos

Os dados obtidos nessa pesquisa contribuem para orientar medidas de caráter preventivo e de controle de DTA a serem implementados pelos serviços de Vigilância em Saúde.

\section{Agradecimentos}

Agradecemos ao LACEN e a $2^{\text {a }}$ Regional de Saúde do Estado do Paraná pelo apoio durante toda a coleta de dados para elaboração do presente trabalho.

\section{Referências}

ALCOCER, I. R. Sorotipagem, fagotipagem, caracterização molecular de cepas de Salmonella spp. e avaliação epidemiológica de surtos ocorridos no Paraná de 1999 a 2004. 2004. 218 
f. Tese (Doutorado em Ciência de Alimentos)

- Departamento de Tecnologia de Alimentos e Medicamentos, Centro de Ciências Agrárias, Universidade Estadual de Londrina, Londrina.

AMSON, G. V.; HARACEMIV, S. M. C.; MASSON, M. L. Levantamento de dados epidemiológicos relativos a ocorrências/ surtos de doenças transmitidas por alimentos (DTAs) no estado do Paraná - Brasil, no período de 1978 a 2000. Revista Ciência e Agrotecnologia, Lavras, v. 30, n. 6, p. 1139-1145, nov./dez. 2006.

BRASIL. Ministério da Saúde. Portaria n ${ }^{\circ} 1.565$, de 26 agosto de 1994. Define o Sistema Nacional de Vigilância Sanitária e sua abrangência, esclarece a competência das três esferas de governo e estabelece as bases para a descentralização da execução dos serviços e ações de vigilância em saúde no âmbito do Sistema Único de Saúde. Diário Oficial [da] República Federativa do Brasil, Poder Executivo, Brasília, DF, 29 ago. 1994.

BRASIL. Ministério da Saúde. Vigilância epidemiológica das doenças transmitidas por alimentos no Brasil 1999 - 2004. Boletim Eletrônico Epidemiológico, Brasília, DF, n. 6, p. 1, 2005.

EDUARDO, M. B. P.; KATSUYA, E. M.; BASSIT, N. P. Características dos surtos de doenças transmitidas por alimentos associados a restaurantes no estado de São Paulo, 1999-2002. Revista de Higiene Alimentar, São Paulo, v. 17, n. 104-105, p. 60, jan./fev. 2003.

HOBBS, B. C.; ROBERTS, D. Toxinfecções e controle higienico-sanitário de alimentos. São Paulo: Varela, 1999. 376 p.

LARIA, S. T.; FURLANETTO, S. M. P.; CAMPOS, M. L. C. Staphylococcus aureus in the anterior portion of nasal fossae of hospital food handlers, São Paulo, Brazil, 1976. Revista de Saúde Pública, São Paulo, v. 14, n. 1, p. 93-100, mar. 1980.

LOPES, R. L. T. Fontes de contaminação de alimentos. Belo Horizonte: CETEC, 2007.
OPAS. Guía veta: guía para el establecimiento de sistemas de vigilancia epidemiológica de enfermedades transmitidas por alimentos $y$ la investigación de brotes de toxi-infecciones alimentarias. Buenos Aires: OPAS, 2001.

PARANÁ. Secretaria de Estado da Saúde do Paraná. Surto alimentar. Disponível em: <http:// www.saude.pr.gov.br/CSA/Surto_alimentar/index. htm>. Acesso em: 27 dez. 2011.

PARANÁ. Secretaria de Saúde. Regionais de Saúde. Disponível em: <http://www.sesa.pr.gov.br/ modules/conteudo/conteudo.php? conteudo $=2752>$. Acesso em: 22 jan. 2012a.

PARANÁ. Secretaria de Saúde. Regionais SESA - $2^{a} R S-C u r i t i b a$. Disponível em: <http:// www.sesa.pr.gov.br/modules/conteudo/conteudo. php? conteudo=2754>. Acesso em: 22 jan. $2012 \mathrm{~b}$.

SANTA CATARINA. Secretaria de Estado da Saúde de Santa Catarina. Manual de orientação para investigação em surtos de DTA. Florianópolis: DIVE, 2006.

SILVA, M. C. D.; RAMALHO, L. S.; FIGUEIREDO, E. T. Salmonella sp. em ovos e carcaças de frango in natura comercializadas em Maceió, AL. Revista de Higiene Alimentar, São Paulo, v. 18, n. 121, p. 80-84, out. 2004.

SNYDER, J. R. O. P. HACCP: an industry safety self-control program (part VI). Dairy, Food and Environmental Sanitation, Iowa, v. 12, n. 6, p. 362365, 1992.

SOARES, A. G.; OLIVEIRA, A. G. M.; FONSECA, M. J. O.; JÚNIOR, M. F. Boas práticas de manipulação em bancos de alimentos. Rio de Janeiro: Embrapa Agroindústria de Alimentos, 2006.

Recebido em: 03 de setembro de 2012. Aceito em: 06 de dezembro de 2012. 